\begin{tabular}{|c|c|c|}
\hline & Int.J.Curr.Microbiol.App.Sci (2016) 5(9): 139-154 & \multirow{4}{*}{ 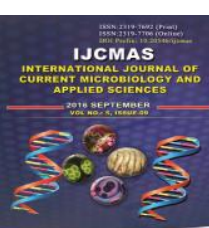 } \\
\hline & \multirow{4}{*}{$\begin{array}{l}\text { International Journal of Current Microbiology and Applied Sciences } \\
\text { ISSN: 2319-7706 Volume } 5 \text { Number } 9 \text { (2016) pp. 139-154 } \\
\text { Journal homepage: http://www.ijcmas.com }\end{array}$} & \\
\hline & & \\
\hline EXCELLENT & & \\
\hline PUBLISHERS & & Imww.jicmas. \\
\hline
\end{tabular}

Original Research Article

http://dx.doi.org/10.20546/ijcmas.2016.509.016

\title{
Foliar Biochemical Approaches in Ten Sugarcane (Saccharum officinarum L.) Cultivars
}

\author{
Dildar Husain ${ }^{1}$, Anoop Kumar ${ }^{2}$ and Zafar Abbas ${ }^{2 *}$ \\ ${ }^{1}$ Department of Botany, Faculty of Science, Maulana Azad Institute of Humanities, Science \\ and Technology, Mahmudabad,Sitapur-261203,U.P, India \\ ${ }^{2}$ P.G. Department of Botany, G.F. College (M.J.P. Rohilkhand University), \\ Shahjahanpur-U.P.- 242001, India \\ *Corresponding author
}

\section{Keywords \\ Sugarcane, rice bran, pyridoxine, starch, amylase activity.}

\begin{tabular}{l}
\hline Article Info \\
\hline Accepted: \\
08 August 2016 \\
Available Online: \\
10 September 2016
\end{tabular}

\section{A B S T R A C T}

A field experiment was conducted during 2007-08 (spring planting) at Agricultural farm of G.F. College, Shahjahanpur U.P. to study the effect of soil applied $(0,10,20,40$ and 60 $\mathrm{kg} / \mathrm{ha}$ ) rice bran alongwith pre-sowing soaking of setts in $0.03 \%$ pyridoxine solution in ten sugarcane (Saccharum officinarum L.) (CoS 8436, CoS 95255, CoS 96268, CoSe 98231, CoSe 01235, CoS 01424, CoSe 95422, UP 097, CoSe 92423 and CoS 96275) cultivars in 4 $\mathrm{x} 2.5 \mathrm{~m}$ plots each treatment replicated thrice in a factorial randomised block design. The soil was sandy loam, $\mathrm{pH} 7.10$, E.C. $0.30 \mathrm{dS} / \mathrm{m}$, Organic carbon $0.25 \%$, available N 150 $\mathrm{kg} / \mathrm{ha}$. available $\mathrm{P} 10.20 \mathrm{~kg} / \mathrm{ha}$ and available $\mathrm{K} 120 \mathrm{~kg} / \mathrm{ha}$. A uniform basal dose of $150 \mathrm{~kg}$ $\mathrm{N}, 60 \mathrm{~kg} \mathrm{P}$ and $80 \mathrm{~kg} \mathrm{~K} / \mathrm{ha}$ was applied to each bed before sowing. Nitrogen was given in $(1 / 3+1 / 3+1 / 3)$ split doses as top dressing.Urea, monocalcium single super phosphate and muriate of potash were used as perspective sources of NPK. Third leaf was selected for the biochemical assays for fresh tissue material at pre-monsoon (120 DAP), post-monsoon (210 DAP), early ripenning (270 DAP), late ripenning (330 DAP) and at maturity (360 DAP) of the crop. The seed rate was $(90 \times 45 \mathrm{~cm})$ row to plant spacing. Weeding was done when required during the course of growth of plants. Standard agronomical practices were followed of the crop. The crop was sown on 24 Feb 2007 and harvested for early varieties on 20 January 2008 and 1 March 2008 for late varieties (DAP = Days after planting). Authentic seeds of sugarcane (Saccharum officinarum L.) varieties were obtained from Division of Sugarcane Breeding, U.P. Council of Sugarcane Research, Shahjahanpur. U.P. A significant increase in leaf starch content was noted as a result of soil applied rice bran as well as $0.03 \%$ pyridoxine soaking upto post monsoon (210 days) stage. The later two final stages showed a reverse trend as compared to control. CoSe 92423 showing mostly highest values for leaf starch content at all growth stages as compared to other cultivars showing a high source potential. As far as the interaction effect was concerned $0.03 \%$ pyridoxine soaking x CoSe 92423 gave significantly highest value upto post monsoon (210 days) stage and lowest value in the later final stages followed by the interaction $40 \mathrm{~kg} / \mathrm{ha}$ soil-applied rice bran $\mathrm{x}$ CoSe 92423. The lower values in the last two stages shows conversion of carbohydrates into sugars for the storage. There was an increase in the level of amylase content significantly upto maturity stage. The impact of $0.03 \%$ pyridoxine sett soaking was maximum for this trait followed by $40 \mathrm{~kg} / \mathrm{ha}$ soil applied rice bran foliar biochemical analysis. CoSe 92423 responded most and gave maximum amylase activity at all stages of growth compared to other cultivars seems to be linked directly with sucrose accumulation in the cane. 


\section{Introduction}

Sugarcane is one of the world's economically most important cultivated crop (Rehim and Espig, 1991). It is the chief source of centrifugal sugar in the world and it contributed nearly 60 per cent world sugar production (Gupta et al. 2004). It is one of the most important cash crops of tropics and sub-tropics in India. It plays a pivotal role in both agricultural and industrial economy of our country. India is one of the largest producers of sugar and is in neck-to-neck race with Brazil for first position. The Indian share in sugar production is about 13 per cent of the world and 41 per cent of Asia (Chinnasamy and Jayanthi, 2004). In India, sugarcane is grown under different agro climatic conditions and occupies about 22 per cent area (4.4 m ha) of the GCA with an average productivity of $68.2 \mathrm{t} / \mathrm{ha}$ (Patel et al. 2008). The area under sugarcane cultivation in Uttar Pradesh (U.P.) alone is about $48 \%$ of India. But from the productivity point of view it is lagging behind. It has two main reasons one is the agro-climatic conditions which is not up to the desired level and second one is the lack of cane production technology management. As far as planting technology is concerned in Punjab, Haryana and Western U.P., the majority of farmers mostly prefer late planting i.e. sugarcane cultivation after wheat crop harvesting. Specially, in Western U.P. about $60 \%$ area is covered under this process which adversly effect the productivity of sugarcane due to poor tillers formation on account of early monsoon rains and moreover the emergence of plenty of weeds. It had been established that 32 to $35 \%$ deterioration in yield is due to late planting adoptation against autumn and spring planting. Sundra (1998) reported that summer planted crop gives very low yield, particularly due to poor germination and tillering because of extremely high temperature coupled with moisture stress. The cane productivity declines by 30 to $50 \%$ if planting is delayed upto end of April or early May (Verma, 2004 and Singh et al., 2008). It is also well known that the autumn (October) planted cane yields $20 \%$ more than the spring (February-March) planted cane, and matures earlier but it needs $30 \%$ more time. Infact, the first Green Revolution, though mostly unsung, took place in sugarcane in 1920s, when the new interspecific hybrids (Saccharum officinarum x $S$. spontaneum) starting with cultivar 'Co205' - yielded 21/2 times more than the traditional canes (varieties belonging to $S$. barberi and $S$. sinense, mostly used for sugar making). This success of new hybrids may be gauged from the fact that within 10 years number of sugar mills in India rose from 20 in 1920 to 110 in 193334. The architect of this unsung revolution, Dr. T.S. Venkataraman, the then Director of Sugarcane Breeding Centre, Coimabtore, was knighted in 1942 by the then imperial government. Today, the cultivated sugarcane is no longer S. officinarum but a Saccharum hybrid complex involving different species of saccharum and allied genera. The process of producing cane hybrids using $S$. officinarum, as female parent is called 'Nobilization of Sugarcane'. The Coimbatore-bred hybrid canes (Popularly known as 'Co canes') supported the sugar industries of different countries including those of the United States of America, South Africa and many others. In India, before the advent of these hybrids more than $80 \%$ sugar was produced in the states of IndoGangetic plains. The development of hybrids gradually increased the cane cultivation in the tropical states. Sugarcane is considered a tropical plant and grows well in that environment. Thus tropical states with longer growing period of sugarcane always have more cane yield than the subtropical states, where the growing period for sugarcane is shorter due to climatic extremes (very high temperature during the 
formative phase and very low temperature during the maturity phase). The natural disparity may be minimised through appropriate agronomic interventions. Hence, the choice of a suitable cultivar, season and adoption of viable crop management strategies play a decisive role in augmenting sugarcane production.

Funk in 1912 was the first to isolate an amine from rice husk and polishing that alleviated the symptoms of disease "beriberi". He also proposed the generic term vitamine for it (Lehninger, 1978). The function of different vitamins in animals can be easily studied, by merely removing a particular vitamin from the diet. This study is, however, difficult in plants, since they synthesize vitamins themselves but the concentration level may not be enough to carry out regulatory and other biochemical processes at optimum level, therefore, externally applied vitamins to plants increase growth and yield potential in different crop plants (Abreg, 1961; Bonner and Bonner, 1948 and Kishor, 2006; Kishor et al., 2006; Kumar et al., 2009 a,b, Samiullah et al., 1985). Moreover, Bonner and Greene (1939) maintained that a plant would respond to exogenous supply of the vitamins only if its endogenous vitamin levels were low. The hypothesis has been confirmed in the authors laboratory were highly encouraging for peppermint crop (Kishor, 2006 and Kishor et al., 2006). With the new high yielding different genotypes of sugarcane available for research, the present author decided to test these from the point of view of (i) growth characters and correlations between biochemical alterations under the influence of soil applied rice bran (a natural source of pyridoxine) alongwith pyridoxine soaked sett as well as foliarlyapplied aqueous rice bran extract on ripening and maturity of sugarcane cultivars, since, the growth and storage are reciprocally related presumably because of competition for the available photosynthate (Hatch et al., 1963).

The present study was the effect of different doses of soil - applied rice bran $(0,10,20$, 40 and $60 \mathrm{~kg} / \mathrm{ha}$ ) alongwith pre-sowing $0.03 \%$ pyridoxine soaked sett on leaf starch content \& amylase activity at different stages (pre-monsoon, post-monsoon, early ripening, late ripening and maturity) of development in ten selected sugarcane cultivars.

\section{Materials and Methods}

This field experiment was conducted during 2007-08 (spring planting) at AgriculturaI farm of G.F. College, Shahjahanpur, U.P., India.

The aim of the experiment was to establish the optimum dose of soil-applied rice bran alongwith pre-sowing soaking of sets in $0.03 \%$ pyridoxine solution of ten sugarcane varieties (five early, $\operatorname{CoS} 8436, \operatorname{CoS} 95255$, CoS 96268, CoSe 98231 and CoSe 01235 and five late, CoS 01424, CoSe 95422, UP 097, CoSe 92423 and $\mathrm{CoS}$ 96275) taking into consideration leaf starch content and amylase activity at different growth stages.

Thus, different doses of soil applied rice bran $(0,10,20,40$ and $60 \mathrm{~kg} / \mathrm{ha})$ were supplied in soil below the sets at the time of sowing as well as pre-sowing soaking of sets is $0.03 \%$ pyridoxine solution for four hours in $4 \times 2.5 \mathrm{~m}$ plots. All the treatments were replicated thrice. Ten sugarcane varieties (five early, $\mathrm{CoS}$ 8436, CoS 95255, CoS 96268, CoSe 98231 and CoSe 01235 and five late, $\mathrm{CoS}$ 01424, CoSe 95422, UP 097, CoSe 92423 and CoS 96275) were tested. A uniform basal dose of $150 \mathrm{~kg} \mathrm{~N}, 60 \mathrm{~kg} \mathrm{P}$ and $80 \mathrm{~kg} \mathrm{~K} / \mathrm{ha}$ was applied to each bed before sowing, Nitrogen was given in $(1 / 3+1 / 3+$ 
1/3) split doses as top dressing. Urea, monocalcium single superphosphate and muriate of potash were used as prespective sources of NPK. Samplings for leaf starch content and amylase activity and third leaf selected for biochemical estimations were done randomly at pre-monsoon (120 DAP), post-monsoon (210 DAP), early ripening (270 DAP), late ripening (330 DAP) and at maturity (360 DAP) of the crop. The seed rate was $(90 \times 45)$ row to plant spacing. Weeding was done when required during the course of growth of plants. Agronomical practices were employed according to crop. The crop was sown on 24 Feb. 2007 and harvested for early varieties on 20 January 2008 and 1 March 2008 for late varieties. (DAP $=$ Days after planting).

Methods of starch and amylase activity :

The fresh third leaf $(5 \mathrm{~g})$ sample collected after noting growth attributes from the plant were homogenised to a fine paste, in a chilled mortar with $10 \mathrm{ml}$. of citrate buffer (pH 5.0). The homogenised samples were then centrifuged (IEC refrigerated. PR-2) at 2500 r.p.m. for 15 minutes at $4^{\circ} \mathrm{C}$. The supernatant (enzyme extract) were used for enzyme assay.

\section{Amylase assay}

The amylase activity was determined following the method of Paleg (1960). 1.0 $\mathrm{ml}$. of enzyme preparation was incubated at $30^{\circ} \mathrm{C}$ with $1.0 \mathrm{ml}$. of citrate buffer and $5 \mathrm{ml}$ of $0.02 \%$ starch solution. The reaction was arrested after 15 minutes by adding $0.2 \mathrm{ml}$ of mercuric chloride $(4 \times 10-4 \quad \mathrm{~N})$. The residual starch was read in a colorimeter at $600 \mathrm{~nm}$ after adding $0.1 \mathrm{ml}$ of I2KI solution and make up the volume to $10 \mathrm{ml}$. Parallel series without enzymes was also run for each treatment. Residual starch content was determined by standard curve plotted by known dilutions of starch (B.D.H.) solution.
Amylase activity was expressed as mg starch digested/gm fresh weight/hour.

\section{Starch content}

Starch content of the leaf was also determined by (Paleg, 1960) with the supernatent (enzyme extract) collected from fresh leaves. To $5.0 \mathrm{ml}$ of supernatent added $1.0 \mathrm{ml}$ of $\mathrm{I} 2 \mathrm{KI}$ solution. The intensity of blue colour was read at $600 \mathrm{~nm}$ in a colorimeter. The starch content was calculated by comparing known dilutions of standard starch solution.

\section{Results and Discussion}

It had become abundantly clear in the early forties that besides plant hormones e.g. auxins, gibberellins etc., growth promoting substances included another set of heterogenous organic compounds (Vitamins) with varying modes of action. These compounds are required in small amounts only, and, in a more or less unchanged form, participate at various stages in the labyrinth called metabolism. However, knowledge of the vitamin requirement of plants had to wait the development of organ and tissue culture techniques because, although whole plants are capable of synthesizing them, their organs can not do so separately. Thus, within the intact plant, by virtue of their translocation from the site of synthesis to that of action in plants.

A significant increase in leaf starch content was noted as a result of soil-applied rice bran as well as $0.03 \%$ pyridoxine soaking (T6) upto post mosoon stage (210 days), tables 1 to 5. The later final stages showed a reverse trend as compared to control (Tables 1 to 2). Variety CoSe 92423 (V9) showed mostly highest values for leaf starch content at all growth stages as compared to other cultivars. 
Table.1 Effect of soil-applied rice bran and pre-sowing pyridoxine soaking of sets on leaf starch (\%) at pre-monsoon (120 days) in sugarcane (Sachharum officinarum L.)

(Mean of three replicates)

\begin{tabular}{|c|c|c|c|c|c|c|c|}
\hline \multirow[b]{2}{*}{ Varieties } & \multicolumn{5}{|c|}{ Soil-applied rice bran (kg/ha) } & \multirow{2}{*}{$\begin{array}{c}0.03 \% \\
\text { Pyridoxine } \\
\left(\mathrm{T}_{6}\right) \text { Soaking } \\
\end{array}$} & \multirow[t]{2}{*}{ Mean } \\
\hline & $\mathbf{0}\left(\mathbf{T}_{1}\right)$ & $10\left(T_{2}\right)$ & $20\left(\mathbf{T}_{3}\right)$ & $40\left(T_{4}\right)$ & $60\left(T_{5}\right)$ & & \\
\hline $\operatorname{CoS} 8436\left(\mathrm{~V}_{1}\right)$ & 1.98 & 2.10 & 2.15 & 2.20 & 2.19 & 2.22 & 2.14 \\
\hline $\operatorname{CoS} 95255\left(V_{2}\right)$ & 1.99 & 2.00 & 2.10 & 2.11 & 2.15 & 2.19 & 2.09 \\
\hline $\operatorname{CoS} 96268\left(\mathrm{~V}_{3}\right)$ & 1.89 & 1.89 & 1.95 & 1.99 & 2.10 & 2.20 & 2.00 \\
\hline CoSe $98231\left(\mathrm{~V}_{4}\right)$ & 1.60 & 1.65 & 1.70 & 1.73 & 1.83 & 1.90 & 1.74 \\
\hline CoSe $01235\left(V_{5}\right)$ & 1.65 & 1.71 & 1.79 & 1.85 & 1.91 & 1.98 & 1.82 \\
\hline $\operatorname{CoS} 01424\left(V_{6}\right)$ & 1.66 & 1.75 & 1.77 & 1.89 & 1.99 & 2.10 & 1.86 \\
\hline CoSe $95422\left(V_{7}\right)$ & 1.65 & 1.69 & 1.88 & 1.95 & 2.10 & 2.15 & 1.90 \\
\hline UP $097\left(V_{8}\right)$ & 1.99 & 2.13 & 2.21 & 2.23 & 2.30 & 2.35 & 2.20 \\
\hline CoSe $92423\left(V_{9}\right)$ & 2.10 & 2.30 & 2.40 & 2.50 & 2.65 & 2.85 & 2.47 \\
\hline $\operatorname{CoS} 96275\left(\mathrm{~V}_{10}\right)$ & 1.77 & 1.91 & 1.98 & 2.10 & 2.11 & 2.19 & 2.01 \\
\hline \multirow[t]{2}{*}{ Mean } & 1.83 & 1.91 & 1.99 & 2.06 & 2.13 & 2.21 & \\
\hline & & \multicolumn{3}{|c|}{ CD at $5 \%$} & \multicolumn{2}{|c|}{ F-value } & \\
\hline \multicolumn{2}{|l|}{ Treatments } & \multicolumn{3}{|c|}{0.031} & \multicolumn{2}{|r|}{$*$} & \\
\hline \multicolumn{2}{|l|}{ Varieties } & \multicolumn{3}{|c|}{0.041} & \multicolumn{2}{|r|}{$*$} & \\
\hline \multicolumn{2}{|c|}{ Treatments $\times$ Varieties } & \multicolumn{3}{|c|}{0.100} & \multicolumn{2}{|r|}{$*$} & \\
\hline
\end{tabular}

$*$ = Significant 
Table.2 Effect of soil-applied rice bran and pre-sowing pyridoxine soaking of sets on leaf starch (\%) at post-monsoon (210 days) in sugarcane (Sachharum officinarum L.)

(Mean of three replicates)

\begin{tabular}{|c|c|c|c|c|c|c|c|}
\hline \multirow{2}{*}{ Varieties } & \multicolumn{5}{|c|}{ Soil-applied rice bran (kg/ha) } & \multirow{2}{*}{$\begin{array}{c}0.03 \% \\
\text { Pyridoxine } \\
\left(\mathbf{T}_{6}\right) \text { Soaking } \\
\end{array}$} & \multirow[t]{2}{*}{ Mean } \\
\hline & $\mathbf{0}\left(\mathbf{T}_{1}\right)$ & $10\left(T_{2}\right)$ & $20\left(T_{3}\right)$ & $40\left(T_{4}\right)$ & $60\left(T_{5}\right)$ & & \\
\hline $\operatorname{CoS} 8436\left(\mathrm{~V}_{1}\right)$ & 2.10 & 2.15 & 2.16 & 2.21 & 2.29 & 2.40 & 2.22 \\
\hline $\operatorname{CoS} 95255\left(V_{2}\right)$ & 2.19 & 2.23 & 2.31 & 2.39 & 2.40 & 2.45 & 2.33 \\
\hline $\operatorname{CoS} 96268\left(\mathrm{~V}_{3}\right)$ & 2.15 & 2.19 & 2.30 & 2.35 & 2.39 & 2.49 & 2.31 \\
\hline CoSe $98231\left(V_{4}\right)$ & 1.98 & 1.99 & 2.05 & 2.10 & 2.35 & 2.36 & 2.14 \\
\hline CoSe $01235\left(V_{5}\right)$ & 1.75 & 1.99 & 2.11 & 2.15 & 2.20 & 2.21 & 2.07 \\
\hline $\operatorname{CoS} 01424\left(\mathrm{~V}_{6}\right)$ & 2.10 & 2.35 & 2.36 & 2.40 & 2.50 & 2.55 & 2.38 \\
\hline CoSe $95422\left(V_{7}\right)$ & 2.30 & 2.39 & 2.41 & 2.77 & 2.81 & 2.90 & 2.60 \\
\hline UP $097\left(\mathrm{~V}_{8}\right)$ & 2.45 & 2.60 & 2.66 & 2.69 & 2.80 & 2.95 & 2.69 \\
\hline CoSe $92423\left(\mathrm{~V}_{9}\right)$ & 2.85 & 2.89 & 2.95 & 2.99 & 2.96 & 2.99 & 2.94 \\
\hline $\operatorname{CoS} 96275\left(\mathrm{~V}_{10}\right)$ & 2.35 & 2.40 & 2.41 & 2.50 & 2.66 & 2.71 & 2.51 \\
\hline \multirow[t]{2}{*}{ Mean } & 2.22 & 2.32 & 2.37 & 2.46 & 2.54 & 2.60 & \\
\hline & & \multicolumn{3}{|c|}{ CD at $5 \%$} & \multicolumn{2}{|c|}{ F-value } & \\
\hline \multicolumn{2}{|l|}{ Treatments } & \multicolumn{3}{|c|}{0.023} & \multicolumn{2}{|r|}{$*$} & \\
\hline \multicolumn{2}{|l|}{ Varieties } & \multicolumn{3}{|c|}{0.030} & \multicolumn{2}{|r|}{$*$} & \\
\hline \multicolumn{2}{|c|}{ Treatments $\times$ Varieties } & \multicolumn{3}{|c|}{0.075} & \multicolumn{2}{|r|}{$*$} & \\
\hline
\end{tabular}


Table.3 Effect of soil-applied rice bran and pre-sowing pyridoxine soaking of sets on leaf starch (\%) at early ripening (270 days) in sugarcane (Sachharum officinarum L.)

(Mean of three replicates)

\begin{tabular}{|c|c|c|c|c|c|c|c|}
\hline \multirow{2}{*}{ Varieties } & \multicolumn{5}{|c|}{ Soil-applied rice bran $(\mathrm{kg} / \mathrm{ha})$} & \multirow{2}{*}{$\begin{array}{c}0.03 \% \\
\text { Pyridoxine } \\
\left(\mathbf{T}_{6}\right) \text { Soaking } \\
\end{array}$} & \multirow[t]{2}{*}{ Mean } \\
\hline & $\mathbf{0}\left(\mathbf{T}_{1}\right)$ & $10\left(T_{2}\right)$ & $20\left(T_{3}\right)$ & $40\left(T_{4}\right)$ & $60\left(T_{5}\right)$ & & \\
\hline $\operatorname{CoS} 8436\left(\mathrm{~V}_{1}\right)$ & 0.70 & 0.68 & 0.69 & 0.67 & 0.59 & 0.59 & 0.65 \\
\hline $\operatorname{CoS} 95255\left(\mathrm{~V}_{2}\right)$ & 0.71 & 0.66 & 0.65 & 0.61 & 0.60 & 0.59 & 0.64 \\
\hline $\operatorname{CoS} 96268\left(V_{3}\right)$ & 0.71 & 0.68 & 0.63 & 0.59 & 0.57 & 0.58 & 0.63 \\
\hline CoSe $98231\left(V_{4}\right)$ & 0.69 & 0.63 & 0.61 & 0.55 & 0.55 & 0.54 & 0.60 \\
\hline CoSe $01235\left(V_{5}\right)$ & 0.68 & 0.65 & 0.60 & 0.77 & 0.54 & 0.50 & 0.62 \\
\hline $\operatorname{CoS} 01424\left(\mathrm{~V}_{6}\right)$ & 0.82 & 0.80 & 0.79 & 0.81 & 0.75 & 0.70 & 0.78 \\
\hline CoSe $95422\left(V_{7}\right)$ & 0.84 & 0.79 & 0.80 & 0.70 & 0.80 & 0.79 & 0.79 \\
\hline UP $097\left(V_{8}\right)$ & 0.85 & 0.80 & 0.81 & 0.79 & 0.74 & 0.70 & 0.78 \\
\hline CoSe $92423\left(V_{9}\right)$ & 0.89 & 0.86 & 0.80 & 0.79 & 0.75 & 0.70 & 0.80 \\
\hline $\operatorname{CoS} 96275\left(\mathrm{~V}_{10}\right)$ & 0.89 & 0.80 & 0.78 & 0.71 & 0.69 & 0.70 & 0.76 \\
\hline \multirow[t]{2}{*}{ Mean } & 0.78 & 0.74 & 0.72 & 0.70 & 0.66 & 0.64 & \\
\hline & & \multicolumn{3}{|c|}{ CD at $5 \%$} & \multicolumn{2}{|c|}{ F-value } & \\
\hline \multicolumn{2}{|l|}{ Treatments } & \multicolumn{3}{|c|}{0.035} & \multicolumn{2}{|r|}{$*$} & \\
\hline \multicolumn{2}{|l|}{ Varieties } & \multicolumn{3}{|c|}{0.045} & \multicolumn{2}{|r|}{$*$} & \\
\hline \multicolumn{2}{|c|}{ Treatments $\times$ Varieties } & \multicolumn{3}{|c|}{0.111} & \multicolumn{2}{|r|}{ NS } & \\
\hline
\end{tabular}

$*$ = Significant, NS = Non-significant 
Table.4 Effect of soil-applied rice bran and pre-sowing pyridoxine soaking of sets on leaf starch (\%) at late ripening (330 days) in sugarcane (Sachharum officinarum L.)

(Mean of three replicates)

\begin{tabular}{|c|c|c|c|c|c|c|c|}
\hline \multirow{2}{*}{ Varieties } & \multicolumn{5}{|c|}{ Soil-applied rice bran $(\mathrm{kg} / \mathrm{ha})$} & \multirow{2}{*}{\begin{tabular}{|c|}
$0.03 \%$ \\
Pyridoxine \\
$\left(\mathrm{T}_{6}\right)$ Soaking \\
\end{tabular}} & \multirow[t]{2}{*}{ Mean } \\
\hline & $\mathbf{0}\left(\mathbf{T}_{\mathbf{1}}\right)$ & $10\left(T_{2}\right)$ & $20\left(T_{3}\right)$ & $40\left(T_{4}\right)$ & $60\left(T_{5}\right)$ & & \\
\hline $\operatorname{CoS} 8436\left(\mathrm{~V}_{1}\right)$ & 0.51 & 0.48 & 0.43 & 0.40 & 0.39 & 0.39 & 0.43 \\
\hline $\operatorname{CoS} 95255\left(\mathrm{~V}_{2}\right)$ & 0.48 & 0.46 & 0.44 & 0.43 & 0.41 & 0.40 & 0.44 \\
\hline $\operatorname{CoS} 96268\left(\mathrm{~V}_{3}\right)$ & 0.46 & 0.43 & 0.40 & 0.40 & 0.39 & 0.39 & 0.41 \\
\hline CoSe $98231\left(V_{4}\right)$ & 0.46 & 0.45 & 0.40 & 0.38 & 0.38 & 0.38 & 0.41 \\
\hline CoSe $01235\left(V_{5}\right)$ & 0.42 & 0.40 & 0.39 & 0.38 & 0.37 & 0.36 & 0.39 \\
\hline $\operatorname{CoS} 01424\left(\mathrm{~V}_{6}\right)$ & 0.58 & 0.50 & 0.48 & 0.42 & 0.40 & 0.38 & 0.46 \\
\hline CoSe $95422\left(V_{7}\right)$ & 0.56 & 0.49 & 0.46 & 0.41 & 0.40 & 0.40 & 0.45 \\
\hline UP $097\left(V_{8}\right)$ & 0.50 & 0.48 & 0.42 & 0.40 & 0.39 & 0.41 & 0.43 \\
\hline CoSe $92423\left(V_{9}\right)$ & 0.59 & 0.53 & 0.50 & 0.47 & 0.40 & 0.37 & 0.48 \\
\hline $\operatorname{CoS} 96275\left(\mathrm{~V}_{10}\right)$ & 0.54 & 0.50 & 0.49 & 0.48 & 0.45 & 0.42 & 0.48 \\
\hline \multirow{2}{*}{ Mean } & 0.51 & 0.47 & 0.44 & 0.42 & 0.40 & 0.39 & \\
\hline & & \multicolumn{3}{|c|}{ CD at $5 \%$} & \multicolumn{2}{|c|}{ F-value } & \\
\hline \multicolumn{2}{|l|}{ Treatments } & \multicolumn{3}{|c|}{0.0081} & \multicolumn{2}{|r|}{$*$} & \\
\hline \multicolumn{2}{|l|}{ Varieties } & \multicolumn{3}{|c|}{0.0105} & \multicolumn{2}{|r|}{$*$} & \\
\hline \multicolumn{2}{|c|}{ Treatments $\times$ Varieties } & \multicolumn{3}{|c|}{0.0259} & \multicolumn{2}{|r|}{$*$} & \\
\hline
\end{tabular}

$$
*=\text { Significant }
$$


Table.5 Effect of soil-applied rice bran and pre-sowing pyridoxine soaking of sets on leaf starch (\%) at maturity (360 days) in sugarcane (Sachharum officinarum L.)

(Mean of three replicates)

\begin{tabular}{|c|c|c|c|c|c|c|c|}
\hline \multirow{2}{*}{ Varieties } & \multicolumn{5}{|c|}{ Soil-applied rice bran (kg/ha) } & \multirow{2}{*}{\begin{tabular}{|c|}
$0.03 \%$ \\
Pyridoxine \\
$\left(\mathbf{T}_{6}\right)$ Soaking \\
\end{tabular}} & \multirow[t]{2}{*}{ Mean } \\
\hline & $\mathbf{0}\left(\mathbf{T}_{1}\right)$ & $10\left(T_{2}\right)$ & $20\left(\mathrm{~T}_{3}\right)$ & $40\left(T_{4}\right)$ & $60\left(T_{5}\right)$ & & \\
\hline $\operatorname{CoS} 8436\left(\mathrm{~V}_{1}\right)$ & 0.00 & 0.00 & 0.00 & 0.00 & 0.00 & 0.00 & 0.00 \\
\hline $\operatorname{CoS} 95255\left(\mathrm{~V}_{2}\right)$ & 0.00 & 0.00 & 0.00 & 0.00 & 0.00 & 0.00 & 0.00 \\
\hline $\operatorname{CoS} 96268\left(\mathrm{~V}_{3}\right)$ & 0.00 & 0.00 & 0.00 & 0.00 & 0.00 & 0.00 & 0.00 \\
\hline CoSe $98231\left(\mathrm{~V}_{4}\right)$ & 0.00 & 0.00 & 0.00 & 0.00 & 0.00 & 0.00 & 0.00 \\
\hline CoSe $01235\left(V_{5}\right)$ & 0.00 & 0.00 & 0.00 & 0.00 & 0.00 & 0.00 & 0.00 \\
\hline $\operatorname{CoS} 01424\left(\mathrm{~V}_{6}\right)$ & 0.53 & 0.50 & 0.49 & 0.48 & 0.46 & 0.40 & 0.48 \\
\hline CoSe $95422\left(V_{7}\right)$ & 0.50 & 0.48 & 0.46 & 0.42 & 0.40 & 0.38 & 0.44 \\
\hline UP $097\left(V_{8}\right)$ & 0.53 & 0.49 & 0.47 & 0.45 & 0.41 & 0.37 & 0.45 \\
\hline CoSe $92423\left(\mathrm{~V}_{9}\right)$ & 0.56 & 0.49 & 0.43 & 0.41 & 0.40 & 0.39 & 0.45 \\
\hline $\operatorname{CoS} 96275\left(\mathrm{~V}_{10}\right)$ & 0.49 & 0.43 & 0.40 & 0.39 & 0.41 & 0.37 & 0.42 \\
\hline \multirow[t]{2}{*}{ Mean } & 0.26 & 0.24 & 0.23 & 0.22 & 0.21 & 0.19 & \\
\hline & & \multicolumn{3}{|c|}{ CD at $5 \%$} & \multicolumn{2}{|c|}{ F-value } & \\
\hline \multicolumn{2}{|l|}{ Treatments } & \multicolumn{3}{|c|}{0.0077} & \multicolumn{2}{|r|}{$*$} & \\
\hline \multicolumn{2}{|l|}{ Varieties } & \multicolumn{3}{|c|}{0.0099} & \multicolumn{2}{|r|}{$*$} & \\
\hline \multicolumn{2}{|c|}{ Treatments $\times$ Varieties } & \multicolumn{3}{|c|}{0.0244} & \multicolumn{2}{|r|}{$*$} & \\
\hline
\end{tabular}

$$
* \text { = Significant }
$$


Table.6 Effect of soil-applied rice bran and pre-sowing pyridoxine soaking of sets on leaf amylase (mg/h/mg protein) at pre-monsoon (120 days) in sugarcane (Sachharum officinarum L.)

(Mean of three replicates)

\begin{tabular}{|c|c|c|c|c|c|c|c|}
\hline \multirow{2}{*}{ Varieties } & \multicolumn{5}{|c|}{ Soil-applied rice bran $(\mathrm{kg} / \mathrm{ha})$} & \multirow{2}{*}{$\begin{array}{c}0.03 \% \\
\text { Pyridoxine } \\
\left(\mathbf{T}_{6}\right) \text { Soaking }\end{array}$} & \multirow[t]{2}{*}{ Mean } \\
\hline & $\mathbf{0}\left(\mathbf{T}_{1}\right)$ & $10\left(T_{2}\right)$ & $20\left(\mathbf{T}_{3}\right)$ & $40\left(T_{4}\right)$ & $60\left(T_{5}\right)$ & & \\
\hline $\operatorname{CoS} 8436\left(\mathrm{~V}_{1}\right)$ & 28.01 & 25.30 & 27.10 & 28.01 & 29.10 & 30.10 & 27.94 \\
\hline $\operatorname{CoS} 95255\left(\mathrm{~V}_{2}\right)$ & 35.50 & 37.10 & 38.90 & 39.55 & 39.50 & 40.90 & 38.58 \\
\hline $\operatorname{CoS} 96268\left(V_{3}\right)$ & 38.60 & 36.10 & 35.25 & 38.30 & 39.11 & 40.10 & 37.91 \\
\hline CoSe $98231\left(V_{4}\right)$ & 36.50 & 35.90 & 38.10 & 39.10 & 39.90 & 41.10 & 38.43 \\
\hline CoSe $01235\left(V_{5}\right)$ & 25.90 & 29.10 & 30.35 & 32.60 & 35.66 & 37.75 & 31.89 \\
\hline $\operatorname{CoS} 01424\left(\mathrm{~V}_{6}\right)$ & 39.00 & 38.10 & 39.90 & 41.60 & 45.10 & 46.20 & 41.65 \\
\hline CoSe $95422\left(V_{7}\right)$ & 41.70 & 42.10 & 43.95 & 43.99 & 46.10 & 49.90 & 44.62 \\
\hline UP $097\left(V_{8}\right)$ & 45.10 & 46.30 & 47.60 & 44.30 & 44.40 & 47.90 & 45.93 \\
\hline CoSe $92423\left(\mathrm{~V}_{9}\right)$ & 49.90 & 49.95 & 51.80 & 53.60 & 55.85 & 54.90 & 52.67 \\
\hline $\operatorname{CoS} 96275\left(\mathrm{~V}_{10}\right)$ & 39.30 & 40.25 & 42.40 & 45.24 & 46.36 & 47.64 & 43.53 \\
\hline \multirow[t]{2}{*}{ Mean } & 37.95 & 38.02 & 39.54 & 40.63 & 42.11 & 43.65 & \\
\hline & & \multicolumn{3}{|c|}{ CD at $5 \%$} & \multicolumn{2}{|c|}{ F-value } & \\
\hline \multicolumn{2}{|l|}{ Treatments } & \multicolumn{3}{|c|}{2.09} & \multicolumn{2}{|r|}{$*$} & \\
\hline \multicolumn{2}{|l|}{ Varieties } & \multicolumn{3}{|c|}{2.717} & \multicolumn{2}{|r|}{$*$} & \\
\hline \multicolumn{2}{|c|}{ Treatments $\times$ Varieties } & \multicolumn{3}{|c|}{6.65} & \multicolumn{2}{|r|}{ NS } & \\
\hline
\end{tabular}

$*$ = Significant, $\mathrm{NS}=$ Non-significant 
Table.7 Effect of soil-applied rice bran and pre-sowing pyridoxine soaking of sets on leaf amylase ( $\mathrm{mg} / \mathrm{h} / \mathrm{mg}$ protein) at post-monsoon (210 days) in sugarcane (Sachharum officinarum L.)

(Mean of three replicates)

\begin{tabular}{|c|c|c|c|c|c|c|c|}
\hline \multirow{2}{*}{ Varieties } & \multicolumn{5}{|c|}{ Soil-applied rice bran (kg/ha) } & \multirow{2}{*}{$\begin{array}{c}0.03 \% \\
\text { Pyridoxine } \\
\left(\mathrm{T}_{6}\right) \text { Soaking }\end{array}$} & \multirow[t]{2}{*}{ Mean } \\
\hline & $\mathbf{0}\left(\mathbf{T}_{1}\right)$ & $10\left(T_{2}\right)$ & $20\left(T_{3}\right)$ & $40\left(T_{4}\right)$ & $60\left(T_{5}\right)$ & & \\
\hline $\operatorname{CoS} 8436\left(\mathrm{~V}_{1}\right)$ & 30.60 & 32.40 & 33.60 & 36.50 & 37.20 & 38.90 & 34.87 \\
\hline $\operatorname{CoS} 95255\left(V_{2}\right)$ & 39.40 & 41.20 & 44.20 & 45.10 & 46.30 & 48.10 & 44.05 \\
\hline $\operatorname{CoS} 96268\left(V_{3}\right)$ & 39.50 & 40.90 & 41.90 & 43.70 & 44.65 & 47.75 & 43.07 \\
\hline CoSe $98231\left(V_{4}\right)$ & 38.10 & 39.82 & 40.90 & 42.70 & 41.90 & 44.80 & 41.37 \\
\hline CoSe $01235\left(V_{5}\right)$ & 28.60 & 30.80 & 32.40 & 34.75 & 36.55 & 38.10 & 33.53 \\
\hline $\operatorname{CoS} 01424\left(\mathrm{~V}_{6}\right)$ & 42.30 & 43.10 & 45.10 & 46.20 & 48.30 & 49.90 & 45.82 \\
\hline CoSe $95422\left(V_{7}\right)$ & 45.15 & 48.10 & 49.10 & 50.10 & 49.10 & 51.00 & 48.76 \\
\hline UP $097\left(V_{8}\right)$ & 49.66 & 50.10 & 52.10 & 53.10 & 52.10 & 55.30 & 52.06 \\
\hline CoSe $92423\left(\mathrm{~V}_{9}\right)$ & 53.10 & 55.10 & 54.90 & 56.10 & 56.90 & 57.10 & 55.53 \\
\hline $\operatorname{CoS} 96275\left(\mathrm{~V}_{10}\right)$ & 41.90 & 42.90 & 44.40 & 45.90 & 46.55 & 46.77 & 44.74 \\
\hline \multirow[t]{2}{*}{ Mean } & 40.83 & 42.44 & 43.86 & 45.42 & 45.96 & 47.77 & \\
\hline & & \multicolumn{3}{|c|}{ CD at $5 \%$} & \multicolumn{2}{|c|}{ F-value } & \\
\hline \multicolumn{2}{|l|}{ Treatments } & \multicolumn{3}{|c|}{1.01} & \multicolumn{2}{|r|}{$*$} & \\
\hline \multicolumn{2}{|l|}{ Varieties } & \multicolumn{3}{|c|}{1.31} & \multicolumn{2}{|r|}{$*$} & \\
\hline \multicolumn{2}{|c|}{ Treatments $\times$ Varieties } & \multicolumn{3}{|c|}{3.22} & \multicolumn{2}{|r|}{ NS } & \\
\hline
\end{tabular}

$$
\text { * = Significant, NS = Non-significant }
$$


Table.8 Effect of soil-applied rice bran and pre-sowing pyridoxine soaking of sets on leaf amylase (mg/h/mg protein) at early ripening (270 days) in sugarcane (Sachharum officinarum L.)

(Mean of three replicates)

\begin{tabular}{|c|c|c|c|c|c|c|c|}
\hline \multirow{2}{*}{ Varieties } & \multicolumn{5}{|c|}{ Soil-applied rice bran (kg/ha) } & \multirow{2}{*}{\begin{tabular}{|c|}
$0.03 \%$ \\
Pyridoxine \\
$\left(\mathrm{T}_{6}\right)$ Soaking \\
\end{tabular}} & \multirow[t]{2}{*}{ Mean } \\
\hline & $\mathbf{0}\left(\mathbf{T}_{1}\right)$ & $10\left(T_{2}\right)$ & $20\left(T_{3}\right)$ & $40\left(T_{4}\right)$ & $60\left(T_{5}\right)$ & & \\
\hline $\operatorname{CoS} 8436\left(\mathrm{~V}_{1}\right)$ & 17.10 & 19.40 & 20.10 & 20.90 & 20.99 & 21.50 & 20.00 \\
\hline $\operatorname{CoS} 95255\left(V_{2}\right)$ & 19.55 & 20.70 & 21.65 & 22.80 & 21.60 & 22.90 & 21.53 \\
\hline $\operatorname{CoS} 96268\left(V_{3}\right)$ & 18.30 & 20.30 & 20.90 & 21.35 & 22.40 & 23.45 & 21.12 \\
\hline $\mathrm{CoSe} 98231\left(\mathrm{~V}_{4}\right)$ & 16.90 & 17.40 & 19.20 & 20.10 & 20.50 & 21.90 & 19.33 \\
\hline CoSe $01235\left(V_{5}\right)$ & 15.30 & 16.30 & 18.70 & 19.50 & 20.10 & 21.55 & 18.58 \\
\hline $\operatorname{CoS} 01424\left(\mathrm{~V}_{6}\right)$ & 30.15 & 31.40 & 32.90 & 34.30 & 36.10 & 36.90 & 33.63 \\
\hline CoSe $95422\left(V_{7}\right)$ & 33.60 & 34.40 & 35.30 & 37.40 & 39.30 & 39.90 & 36.65 \\
\hline UP $097\left(V_{8}\right)$ & 39.10 & 40.30 & 41.30 & 42.90 & 44.10 & 44.80 & 42.08 \\
\hline CoSe $92423\left(V_{9}\right)$ & 40.70 & 42.90 & 42.90 & 44.60 & 45.60 & 48.10 & 44.13 \\
\hline $\operatorname{CoS} 96275\left(\mathrm{~V}_{10}\right)$ & 36.20 & 37.60 & 38.50 & 39.00 & 39.90 & 40.20 & 38.57 \\
\hline \multirow[t]{2}{*}{ Mean } & 26.69 & 28.07 & 29.15 & 30.29 & 31.06 & 32.12 & \\
\hline & & \multicolumn{3}{|c|}{ CD at $5 \%$} & \multicolumn{2}{|c|}{ F-value } & \\
\hline \multicolumn{2}{|l|}{ Treatments } & \multicolumn{3}{|c|}{0.91} & \multicolumn{2}{|r|}{$*$} & \\
\hline \multicolumn{2}{|l|}{ Varieties } & \multicolumn{3}{|c|}{1.17} & \multicolumn{2}{|r|}{$*$} & \\
\hline \multicolumn{2}{|c|}{ Treatments $\times$ Varieties } & \multicolumn{3}{|c|}{2.88} & \multicolumn{2}{|r|}{ NS } & \\
\hline
\end{tabular}

$*$ = Significant, NS = Non-significant 
Table.9 Effect of soil-applied rice bran and pre-sowing pyridoxine soaking of sets on leaf amylase ( $\mathrm{mg} / \mathrm{h} / \mathrm{mg}$ protein) at late ripening (330 days) in sugarcane (Sachharum officinarum L.)

(Mean of three replicates)

\begin{tabular}{|c|c|c|c|c|c|c|c|}
\hline \multirow{2}{*}{ Varieties } & \multicolumn{5}{|c|}{ Soil-applied rice bran (kg/ha) } & \multirow{2}{*}{$\begin{array}{c}0.03 \% \\
\text { Pyridoxine } \\
\left(\mathrm{T}_{6}\right) \text { Soaking }\end{array}$} & \multirow[t]{2}{*}{ Mean } \\
\hline & $\mathbf{0}\left(\mathbf{T}_{1}\right)$ & $10\left(T_{2}\right)$ & $20\left(T_{3}\right)$ & $40\left(T_{4}\right)$ & $60\left(T_{5}\right)$ & & \\
\hline $\operatorname{CoS} 8436\left(\mathrm{~V}_{1}\right)$ & 17.20 & 18.90 & 19.20 & 20.40 & 21.30 & 22.00 & 19.83 \\
\hline $\operatorname{CoS} 95255\left(\mathrm{~V}_{2}\right)$ & 19.85 & 2.20 & 21.30 & 22.40 & 23.40 & 24.50 & 18.94 \\
\hline $\operatorname{CoS} 96268\left(V_{3}\right)$ & 18.80 & 19.30 & 20.30 & 21.30 & 22.90 & 23.70 & 21.05 \\
\hline CoSe $98231\left(V_{4}\right)$ & 16.96 & 17.30 & 18.90 & 19.10 & 20.30 & 21.40 & 18.99 \\
\hline CoSe $01235\left(\mathrm{~V}_{5}\right)$ & 15.80 & 16.30 & 18.10 & 19.50 & 19.60 & 19.90 & 18.20 \\
\hline $\operatorname{CoS} 01424\left(\mathrm{~V}_{6}\right)$ & 40.10 & 40.30 & 42.60 & 44.40 & 45.20 & 46.30 & 43.15 \\
\hline CoSe $95422\left(V_{7}\right)$ & 41.20 & 42.10 & 43.90 & 46.30 & 46.90 & 46.90 & 44.55 \\
\hline UP $097\left(V_{8}\right)$ & 47.30 & 47.10 & 48.30 & 49.40 & 50.10 & 50.90 & 48.85 \\
\hline CoSe $92423\left(\mathrm{~V}_{9}\right)$ & 41.20 & 42.30 & 42.10 & 42.30 & 44.10 & 48.30 & 43.38 \\
\hline $\operatorname{CoS} 96275\left(\mathrm{~V}_{10}\right)$ & 46.10 & 46.80 & 47.10 & 47.90 & 48.40 & 49.30 & 47.60 \\
\hline Mean & 30.45 & 29.26 & 32.18 & 33.30 & 34.22 & 35.32 & \\
\hline & & \multicolumn{3}{|c|}{ CD at $5 \%$} & \multicolumn{2}{|c|}{ F-value } & \\
\hline \multicolumn{2}{|l|}{ Treatments } & \multicolumn{3}{|c|}{0.83} & \multicolumn{2}{|r|}{ * } & \\
\hline \multicolumn{2}{|l|}{ Varieties } & \multicolumn{3}{|c|}{1.07} & \multicolumn{2}{|r|}{$*$} & \\
\hline \multicolumn{2}{|c|}{ Treatments $\times$ Varieties } & \multicolumn{3}{|c|}{2.63} & \multicolumn{2}{|c|}{ NS } & \\
\hline
\end{tabular}

$*=$ Significant, $\mathrm{NS}=$ Non-significant 
Table.10 Effect of soil-applied rice bran and pre-sowing pyridoxine soaking of sets on leaf amylase ( $\mathrm{mg} / \mathrm{h} / \mathrm{mg}$ protein) at maturity (360 days) in sugarcane (Sachharum officinarum L.)

(Mean of three replicates)

\begin{tabular}{|c|c|c|c|c|c|c|c|}
\hline \multirow[b]{2}{*}{ Varieties } & \multicolumn{5}{|c|}{ Soil-applied rice bran (kg/ha) } & \multirow{2}{*}{$\begin{array}{c}0.03 \% \\
\text { Pyridoxine } \\
\left(\mathbf{T}_{6}\right) \text { Soaking }\end{array}$} & \multirow[t]{2}{*}{ Mean } \\
\hline & $\mathbf{0}\left(\mathbf{T}_{1}\right)$ & $10\left(T_{2}\right)$ & $20\left(T_{3}\right)$ & $40\left(T_{4}\right)$ & $60\left(T_{5}\right)$ & & \\
\hline $\operatorname{CoS} 8436\left(V_{1}\right)$ & 0.00 & 0.00 & 0.00 & 0.00 & 0.00 & 0.00 & 0.00 \\
\hline $\operatorname{CoS} 95255\left(V_{2}\right)$ & 0.00 & 0.00 & 0.00 & 0.00 & 0.00 & 0.00 & 0.00 \\
\hline $\operatorname{CoS} 96268\left(V_{3}\right)$ & 0.00 & 0.00 & 0.00 & 0.00 & 0.00 & 0.00 & 0.00 \\
\hline CoSe $98231\left(\mathrm{~V}_{4}\right)$ & 0.00 & 0.00 & 0.00 & 0.00 & 0.00 & 0.00 & 0.00 \\
\hline CoSe $01235\left(V_{5}\right)$ & 0.00 & 0.00 & 0.00 & 0.00 & 0.00 & 0.00 & 0.00 \\
\hline $\operatorname{CoS} 01424\left(\mathrm{~V}_{6}\right)$ & 42.60 & 43.30 & 44.60 & 45.50 & 45.90 & 46.70 & 44.77 \\
\hline $\operatorname{CoSe} 95422\left(\mathrm{~V}_{7}\right)$ & 48.10 & 48.90 & 49.10 & 49.90 & 49.90 & 51.00 & 49.48 \\
\hline UP $097\left(\mathrm{~V}_{8}\right)$ & 47.40 & 47.80 & 48.80 & 49.90 & 49.89 & 50.00 & 48.97 \\
\hline CoSe $92423\left(\mathrm{~V}_{9}\right)$ & 43.90 & 44.10 & 45.60 & 46.45 & 47.10 & 48.40 & 45.93 \\
\hline $\operatorname{CoS} 96275\left(\mathrm{~V}_{10}\right)$ & 47.15 & 48.10 & 49.30 & 49.90 & 50.10 & 50.40 & 49.16 \\
\hline \multirow[t]{2}{*}{ Mean } & 22.92 & 23.22 & 23.74 & 24.17 & 24.29 & 24.65 & \\
\hline & & \multicolumn{3}{|c|}{ CD at $5 \%$} & \multicolumn{2}{|c|}{ F-value } & \\
\hline \multicolumn{2}{|l|}{ Treatments } & \multicolumn{3}{|c|}{1.17} & \multicolumn{2}{|r|}{$*$} & \\
\hline \multicolumn{2}{|l|}{ Varieties } & \multicolumn{3}{|c|}{1.51} & \multicolumn{2}{|r|}{ * } & \\
\hline \multicolumn{2}{|c|}{ Treatments $\times$ Varieties } & \multicolumn{3}{|c|}{3.71} & \multicolumn{2}{|c|}{ NS } & \\
\hline
\end{tabular}

$*=$ Significant, $\mathrm{NS}=$ Non-significant

As far as the interaction effect was concerned, $0.03 \%$ pyridoxine soaking (T6) x CoSe 92423 (V9) gave significantly highest value upto post-mosoon (210 days) stage and lowest value in the later final stages (Tables 1 to 5) followed by a somewhat similar interaction with $40 \mathrm{~kg} / \mathrm{ha}$ soil-applied rice bran (T4) x CoSe 92423 (V9). Leaf amylase activity was significantly affected by soil-applied rice bran as well as $0.03 \%$ pyridoxine soaking (T6) of sets at all stages of growth studied for the sugarcane varieties (Tables 6 to 10). However, the interaction effects at all stages 
for this parameter were found nonsignificant (Tables 6to 10). There was an increase in the level of amylase content upto post-monsoon (210 days) stage with a gradual sharp decrease onwards in the further stages of development, least at maturity (360 days) stage. The effect of different treatments on amylase activity was positively related with an increasing trend at all growth stages, the highest values was recorded $0.03 \%$ pyridoxine soaking (T6) as compared to control (Tables 6 to 10). Among the varieties, CoSe 92423 (V9) gave highest value as compared to other varieties. At maturity (360 days) stage a decreased minimum level in amylase activity was found in CoSe 92423 (V9) as compared to earlier stage (Table 10).

Foliar biochemical components may be regarded as mini imitation of the concentration levels present in the stem tissues. Hence, the foliar biochemical analyses for various components might become a biochemical parameter indicating their status at different growth stages and their associations with sucrose, ripening, maturity and cane yield etc. would be highly valuable (Kumar 2009). There was a gradual significant increase in amylase activity with the advancement in age, highest values were recorded at maturity (360days) stage in late maturing varieties (Table 10) and at late ripening stage (330days) in early maturing cultivars (Table 9). The impact of $0.03 \%$ pyridoxine sett-soaking (T6) was maximum for this trait followedby $40 \mathrm{~kg} / \mathrm{ha}$ soilapplied rice bran (T4) in the foliar biochemical analyses studied with $\mathrm{CoSe}$ 92423 (V9) responded most for all the parameters .The influence of soil-applied rice bran and pyridoxine sett-soaking, indicated that starch, like sugars is an alternate reservoir of carbohydrate for energy and these may be treated as a (biochemical traits) signal for attaining maturity.

\section{Acknowledgement}

The authors are thankful to Dr Aquil Ahmad, Principal, G.F. College Shahjahanpur for the help and encouragements.

\section{References}

Aberg, B. 1961. Vitamins as growth factors in higher plants. In : Encyclopedia of Plant Physiology, ed. W. Ruhland, springer-verlag: Berlin, 14 : 418-449.

Bonner, J. and Bonner, H. 1948. The Bvitamins as plant hormones. Vitam. Horm., 6 : 225-275.

Bonner, J. and Greene, J. 1939. Vitamin-B1 and the growth of green plants. Bot. Gaz., 100: 226-237.

Burstrom, H. 1961. The chemical regulation of growth - General survey. In : Encyclopedia of Plant Physiology, (ed.) W. Ruhlanad, Springer-verlag, Berlin, 14: 324-329.

Chinnasamy, R.T.C. and Jayanthi, C. 2004. Bio-organic nutrient management in sugarcane production, Agric. Rev., 25(3): 201-210.

Gupta, R., Kumar, R. and Tripathi, S.K. 2004. Study on agroclimatic condition and productivity pattern of sugarcane in India. Sugar Tech., 6(3): 141-149.

Hatch, M.D., Sacher, J.A. and Glasziou, K.T. 1963. The sugar accumulation cycle in sugarcane. I. Studies on the enzymes of the cycle. Plant Physiol., 38: $338-343$.

Kishor, B. 2006. Effect of pyridoxine and nitrogen on growth, yield, essential oil and Biochemical components of Mentha piperita. L., under salt stess. Ph.D thesis, M.J.P. Rohilkhand University, Bareilly.

Kishor, B., Kanaujia, S.N. and Abbas, Z. 2006. Effect of vitamin B6 
(Pyridoxine) on the growth, yield, oil content and biochemical components of peppermint Mentha piperita. L. Nat. J. Life Sci., 3(3): 215 - 220.

Kumar, A. 2009. Effect of soil applied rice bran and foliarly applied its extract on carbohydrate and protein metabolism during ripening and maturity in Saccharum officinarum L. Ph.D. thesis M.J.P. Rohilkhand University Bareilly.

Kumar, A., Kumar, A, Kumar, P. and Abbas, Z. 2009b. Effect of soilapplied rice bran on germination percentage and cane yield of ten sugarcane (Saccharum officinarum L.) cultivars. Life Sci. Bull., 6(3): 161164.

Kumar, A., Shukla, S.K. and Abbas, Z. 2009a. Effect of soil-applied rice bran and pyridoxine seaking on sugarcane yield. Sugarcane International (The Journal of Cane Agriculture), (United Kingdom), 27(5): 210 - 211.

Lehninger, R.A. 1978. 'Principles of Biochemistry' (2nd ed.), Worth Publishers, Inc., New York, PP. 335 353.

Paleg, L.G. 1960. Physiological effects of gibberellic acid. II. On starch hydrolysing enzymes of barley endosperm. Plant Physiol., 35: 902 906.

Patel, V.S., Bafna, A.M., Raj, V.C., Colambe, B.N. and Patel, D.D. 2008. Effect of different levels and source of organics on sugarcane (var. CoLK 8001). Indian Sugar, December 2008: $65-70$.

Rehim, S. and Espig, G. 1991. The cultivated plants of tropics and sub_tropics. Priese. Gmbh, Berlin, Germany. pp.66-69.

Samiullah, Ansari, S.A., Afridi, M.M.R.K., and Akhtar, M. 1985. Pyridoxine application enhances nitrate reductase activity and productivity in Vigna radiata. Experientia, 41: 1412-1414.

Singh, J.P., Pal, .S. Ram, S. and Lal, K. 2008. Sugarcane production technology under late spring planting condition for western tract of Uttar Pradesh: At a Glance. Coop. Sugar, 40(4): 57-67.

Sundra, B. 1998. 'Sugarcane cultivation' pp. 27.

Verma, R.S. 2004 'Sugarcane Production Technology in India’. pp. 114-132.

\section{How to cite this article:}

Dildar Husain, Anoop Kumar and Zafar Abbas. 2016. Foliar Biochemical Approaches in Ten Sugarcane (Saccharum officinarum L.) Cultivars. Int.J.Curr.Microbiol.App.Sci. 5(9): 139-154. doi: http://dx.doi.org/10.20546/ijcmas.2016.509.016 